\title{
ABSTRACT
}

\section{Prevalence of HIV among Men who Have Sex with Men (MSM) and Its Associated Factors in Sabah}

Valentine J. G. ${ }^{1 *}$, Diane C. ${ }^{1}$, Chua Y. W. ${ }^{1}$

${ }^{1}$ Sabah Health Department

(HIV/STI/Hep C Unit),

Kota Kinabalu, Sabah,

Malaysia

*Corresponding author's email:

vjapulee@gmail.com

Keywords: HIV infection, men

who have sex with men (MSM),

prevalence, associated factors
Introduction: Men who have sex with men (MSM) is one of the key populations affected by HIV epidemic with infection rates exceeding five percentages. Due to a strict and prohibitive socio-cultural and legal environment, male-tomale sexual practices are often misunderstood and highly stigmatised, thus creating barriers for MSM to access HIV prevention, treatment, care and support services. Objective: This study was to determine the prevalence of HIV infection among MSM. This study also sought to determine factors associated with HIV infection among MSM in Sabah. Methodology: A cross-sectional study was done from April to September 2017 in Sabah. A face-to-face questionnaire interview was conducted among 200 respondents of MSM who were recruited by the Respondent-Driven Sampling (RDS). Sample size was calculated using Epi Info 7 with design effect 2.0. Data was entered and analyzed by SPSS 16.0 using descriptive, univariate and multiple logistic regression analyses. Results: The mean of age was 23.1 (6.08) years with minimum age was 18 years and maximum age was 49 years. Majority was never married with $83.5 \%$ and $51.5 \%$ respondents completed secondary school while $7.5 \%$ received no form of schooling. The prevalence of HIV was 5.0\% (95\% Cl: 2.0\%, 8.0\%). Logistic regression analysis showed that significant risk factors associated with HIV infection among men who have sex with men (MSM) were duration of years of high risk exposure (OR: 1.24; $95 \% \mathrm{Cl}$ : $1.09,1.40 ; p=0.001$ ) and history of anal sex in 
Borneo Journal of Medical Sciences (BJMS), Special Issue, Volume 2, March 2019: 27 - 28

the past one month (OR: $16.07 ; 95 \% \mathrm{Cl}: 2.94$, $87.81 ; p=0.001)$. Conclusion: The prevalence of HIV among MSM was within concentrated HIV epidemic and increasing. Those who had history of anal sex in the past 1 month were 16 times more likely to exhibit HIV and longer duration of high risk exposure was associated with an increased likelihood of exhibiting HIV. Targeted, tailored, and comprehensive interventions are urgently needed to prevent the HIV infection from MSM. 